\title{
Capillary Electrophoresis and Dynamic Light Scattering Studies of Structure and Binding Characteristics of Protein-Polyelectrolyte Complexes
}

\author{
Jeff Y. Gao, ${ }^{\dagger}$ Paul L. Dubin,* and Barry B. Muhoberac \\ Department of Chemistry, Indiana University-Purdue University at Indianapolis, Indianapolis, Indiana 46202
}

Received: December 11, 1997; In Final Form: May 5, 1998

\begin{abstract}
Complex formation between sodium polystyrenesulfonate (NaPSS) and two proteins, bovine serum albumin and $\beta$-lactoglobulin, was studied by dynamic light scattering (DLS) and capillary electrophoresis (CE) over a range of polyelectrolyte molecular weights. We found that the dimensions of the polyelectrolyte chain do not appear to change significantly upon complex formation and that the dependence of complex mobility on its composition is in agreement with a free-draining model. Estimates of intrinsic binding constants, sizes of binding site, and cooperativities in complex formation were obtained by fitting experimental data to the "overlapping binding sites" model. It was found that the cooperativities shown by the binding isotherms are consistent with the dependence of binding density upon NaPSS molecular weights. The latter can be predicted by the overlapping binding sites model for known binding cooperativity, but was not observed experimentally before.
\end{abstract}

\section{Introduction}

The interactions of proteins with polyelectrolytes are important in natural biological systems as well as in biotechnological applications. For example, the binding of proteins to nucleic acids, which are natural polyelectrolytes, is an integral step in gene regulation. ${ }^{1,2}$ Technologically, protein-polyelectrolyte interactions can be utilized for protein separation, ${ }^{3}$ enzyme immobilization, ${ }^{4,5}$ and drug delivery. ${ }^{6,7}$ Current research activity in this area is a reflection of these considerations. Others have dealt with protein-polyelectrolyte binding energetics, ${ }^{1,8,9}$ kinetics, ${ }^{10}$ the model for engagement and recognition, ${ }^{11}$ and various binding models. ${ }^{12,13}$ Our studies focus on the complex structure and the mechanism of its formation.

We have studied complex formation between a number of proteins and a variety of synthetic polyelectrolytes, focusing in particular on the system comprising bovine serum albumin (BSA) and poly(diallyldimethylammonium chloride) (PDADMAC) ${ }^{14-18}$ Depending upon such variables as $\mathrm{pH}$, ionic strength $(I)$, and the stoichiometry of polymer and protein concentrations, complexes may take the form of intrapolymerbound proteins, soluble aggregates, and coacervates or precipitates. Soluble complexes have been of particular interest to us for several reasons. Firstly, soluble complexes appear to be the precursors of more extensive aggregates, so that a knowledge of their structures and formation is prerequisite to understanding the higher-order systems. Secondly, they can be studied with many experimental methods including turbidimetry, ${ }^{16,18}$ dynamic light scattering (DLS), ${ }^{14-16}$ static light scattering, ${ }^{14,17}$ fluorescence spectroscopy, ${ }^{19}$ electrophoretic light scattering, ${ }^{14,15,17,19,20}$ circular dichroism, ${ }^{21}$ and size-exclusion chromatography. ${ }^{22,23}$ However, in many cases, these techniques provide indirect or less quantitative evidence about the degree of protein binding. In this work, we present results of our model systems from capillary electrophoresis and DLS, which provide additional insight into the structure and formation of protein-polyelectrolyte complexes.

\footnotetext{
$\dagger$ Current address: Parke-Davis Pharmaceutical Research, 2800 Plymouth
} Rd., Ann Arbor, MI 48105.
The structure of polyelectrolyte-protein complexes may be described in terms of the conformation of the polyelectrolyte backbone within the complex. Such information may be obtained from the relationship between the diffusion coefficient of the complex $\left(D_{x}\right)$ and its molecular weight $\left(M_{x}\right){ }^{24}$ The determination of $D_{x}$ by, for example, DLS, is generally complicated for such multicomponent samples because the contributions from free polyion and protein may be difficult to separate from those of the complex. Nevertheless, since the intensity of the scattered light is predominantly influenced by the high molecular weight species, measurement of $D_{x}$ is feasible. ${ }^{5}$ Determination of $M_{x}$ has hitherto been yet more difficult. Using a recently developed method, frontal analysis continuous capillary electrophoresis (FACCE), ${ }^{25}$ we can now determine the composition of the complexes, consequently the values of $M_{x}$.

An understanding of complex structure can also be obtained by studying the dependence of its electrophoretic mobility, $\mu_{x}$, on complex composition and on the charge of bound protein. The dependence of $\mu_{x}$ upon solution $\mathrm{pH}$ (i.e., protein charge) for BSA/PDADMAC complexes was previously found to be consistent with a simple relationship based on a free-draining model, ${ }^{14}$ in which $\mu_{x}$ depends on the charges and frictional coefficients of the bound protein and polyelectrolyte: ${ }^{14,15}$

$$
\mu_{x}=\frac{q_{\mathrm{p}}+N q_{\text {pro }}}{f_{\mathrm{p}}+N f_{\text {pro }}}
$$

where $q_{\mathrm{p}}$ and $q_{\mathrm{pro}}$ are the total effective charges on polyion and protein, $f_{\mathrm{p}}$ and $f_{\text {pro }}$ are the frictional coefficients of polyion and protein, and $N$ is the average number of proteins bound per polymer chain. While the effects of ionic strength and specific electrolyte ions are not explicitly considered in eq 1, this simplified treatment is appropriate as long as the supporting electrolyte is invariant. With more precise information about complex composition and mobility in this work, the free-draining model can be evaluated more thoroughly.

The binding of protein to polyelectrolyte may exhibit cooperativity, ${ }^{17}$ which can be identified by analysis of binding 
isotherms using appropriate models. ${ }^{26,27}$ Due to the large size of the proteins (ligands) relative to the polymers (substrates) in this study and the nonspecific nature of the binding process, an appropriate model must consider the effect of overlapping binding sites. The binding model of McGhee and von Hippel ${ }^{12}$ is therefore suitable, particularly if the distribution of binding sites is not complicated by changes in polymer conformation. According to this model, binding due to noninteracting ligands can be expressed as

$$
\frac{v}{L}=K_{\mathrm{b}}(1-n v)\left(\frac{1-n v}{1-(n-1) v}\right)^{n-1}
$$

where $L$ is the free ligand concentration, $K_{\mathrm{b}}$ is the intrinsic binding constant, $n$ is the average size of the binding sites, and $v$ is the binding density defined as the average number of bound ligands per polymer structural unit. When there are interactions between adjacent bound ligands, incorporation of the binding cooperativity, $\omega(<1$ anticooperative, $=1$ noncooperative, $>1$ cooperative), leads to the following McGhee-von Hippel model:

$$
\begin{array}{r}
\frac{v}{L}=K_{\mathrm{b}}(1-n v)\left(\frac{(2 \omega-1)(1-n v)+v-R}{2(\omega-1)(1-n v)}\right)^{n-1} \times \\
\left(\frac{1-(n+1) v+R}{2(1-n v)}\right)^{2}
\end{array}
$$

where

$$
R=\sqrt{\left\{[1-(n+1) v]^{2}+4 \omega v(1-n v)\right\}}
$$

It must be recognized that the cooperativity factor, $\omega$, in the McGhee-von Hippel model describes only interactions between adjacent bound ligands, and any interactions between bound ligands separated by one or more monomer residues are completely ignored. Such a simplified treatment is ill-suited when $\omega$ is used to describe cooperativity for long-range ligand interactions, particularly for anticooperative systems, as an infinite anticooperativity $(\omega=0)$ is merely physically identical to an increase of the size of the binding site by one monomer residue. Therefore, fitting binding data using eqs 3 and 4 for anticooperative systems gives less accuracy in cooperativity than the accuracy in the fitted size of the binding site. Furthermore, quantitative interpretation of binding isotherms with this model can be compromised by the nonideal binding behavior of a real system that deviates from the fixed-size binding site model, particularly at the ends of polymer chains.

Qualitatively, cooperativity in the overlapping binding site model is reflected not only in the shape of the binding isotherm but also in the dependence of binding upon the length of the polymer chain. ${ }^{28}$ However, there has been a lack of experimental observation of such dependence, particularly for proteins binding to a flexible polymer chain. Results reported in this work demonstrate correlation of experimental data with the model.

We have studied the complexation of sodium polystyrenesulfonate (NaPSS) with $\beta$-lactoglobulin and bovine serum albumin, respectively. The two proteins are readily available and well characterized, and monodisperse NaPSS standards of various molecular weights are commercially available. In addition, the use of $\mathrm{CE}$ to study such complexes has been established..$^{25}$

\section{Experimental Section}

a. Materials and Sample Preparation. $\beta$-Lactoglobulin A\&B of molecular weight of $M_{\mathrm{w}}=35500$ was purchased from Sigma (catalog no. L-2506) (St. Louis, MO). Bovine serum albumin (BSA) of $M_{\mathrm{w}}=66000$ was purchased from Boehringer-Mannheim (catalog no. 100062) (Indianapolis, IN). NaPSS with $M_{\mathrm{w}} / M_{\mathrm{n}}<1.1$ and of $M_{\mathrm{w}}$ 's $=710 \mathrm{~K}, 88 \mathrm{~K}, 31 \mathrm{~K}$, and $5.4 \mathrm{~K}$ were obtained from Pressure Chemical Co. (Pittsburgh, PA). The degrees of sulfonation for NaPSS from this source have been reported ${ }^{29}$ to range from $73 \%$ to $88 \%$. NaPSS with $M_{\mathrm{w}} / M_{\mathrm{n}}<1.4$ and of $M_{\mathrm{w}}=465 \mathrm{~K}$ was obtained from Polymer Standard Service (Mainz, Germany). High-purity water with $R \approx 18 \mathrm{M} \Omega \cdot \mathrm{cm}$ generated by a Milli-Q water purification system was used for all solutions. Phosphate buffers were prepared from monobasic and dibasic salts of sodium phosphate obtained from J. T. Baker Chem. Co. (Phillipsburg, NJ).

Samples of protein and NaPSS solutions were prepared, based on volume, from freshly made stock solutions in phosphate buffer of $I=0.05$ and $\mathrm{pH}=6.7$. Before sample preparation, all stock solutions were filtered with $0.45 \mu \mathrm{m}$ Whatman syringefilters (Clifton, NJ) and analyzed spectrophotometrically using published extinction coefficients ${ }^{30}$ at $280 \mathrm{~nm}$ for proteins, and using an extinction coefficient of $1.563 \mathrm{~L} /(\mathrm{g} \cdot \mathrm{cm})$ at $261 \mathrm{~nm}$ obtained in our laboratory for NaPSS. All complex samples were prepared at a fixed concentration of NaPSS $(0.1 \mathrm{~g} / \mathrm{L})$ with $1.85 \mathrm{~g} / \mathrm{L}$ BSA or $0.83 \mathrm{~g} / \mathrm{L} \beta$-lactoglobulin.

b. Methods. Dynamic light scattering was carried out with a DynaPro-801 from Protein Solutions Inc. (Charlottesville, VA), which employs a $25 \mathrm{~mW}$ solid-state $780 \mathrm{~nm}$ laser, a $7 \mu \mathrm{L}$ flow cell, and an avalanche photodiode detector at $90^{\circ}$. Samples were introduced into the flow cell via $0.2 \mu \mathrm{m}$ Anotop filters. Scattering data were analyzed by the method of cumulants. ${ }^{31}$ $\mathrm{CE}$ was carried out at $25 \pm 0.2{ }^{\circ} \mathrm{C}$ with a Beckman P/ACE 5500 (Fullerton, CA) equipped with a UV detector. Capillary selection was based on achieving sufficient resolving power for free and bound protein with maximum efficiency. Although longer capillary and slower electroosmotic flow (EOF) both lead to better resolution, ${ }^{32}$ the latter is more effective in enhancing the resolution in a given time. Therefore, coated and uncoated capillaries with the shortest possible length (total length of 27 $\mathrm{cm}$ and a length from inlet to detection window of $20 \mathrm{~cm}$ ) were used. For $\beta$-lactoglobulin/NaPSS samples, an uncoated capillary from Restek (Bellefonte, PA) with $50 \mu \mathrm{m}$ i.d. provided adequate separation. For BSA/NaPSS samples, a sulfonic-modified capillary (i.d. $=75 \mu \mathrm{m}$ ) with a decreased EOF from ISCO (Lincoln, NE) proved to be better in separating free BSA from the complexes. Separation voltage was $7 \mathrm{kV}$, as determined before. ${ }^{25}$ The mobilities for individual protein and polymer samples were measured using mesityl oxide $(0.2 \% \mathrm{w} / \mathrm{w})$ as a neutral marker to correct for EOF. The mobilities for BSA/ NaPSS complexes were measured by injecting the complex sample directly into protein-free buffer and using the free BSA peak as a secondary mobility standard for EOF correction. Strictly speaking, this method does not prevent the complex from dissociating during the measurement. However, as shown in Figure 1, electropherograms for the BSA/NaPSS sample obtained by FACCE (Figure 1a), an equilibrium method, and by regular sample injection (Figure 1b), a nonequilibrium method, exhibit similar elution time for the free BSA and the BSA/NaPSS complex. Thus, BSA/NaPSS complexes appear to exhibit slow dissociation, so that regular sample injection may be used to study binding stoichiometry ${ }^{33}$ and mobility. ${ }^{34}$ The slightly faster elution of complexes in Figure $1 \mathrm{~b}$ is expected since the average viscosity of the run buffer in direct injection is lower than in FACCE. The mobility of complexes formed between $\beta$-lactoglobulin and NaPSS, however, cannot be 


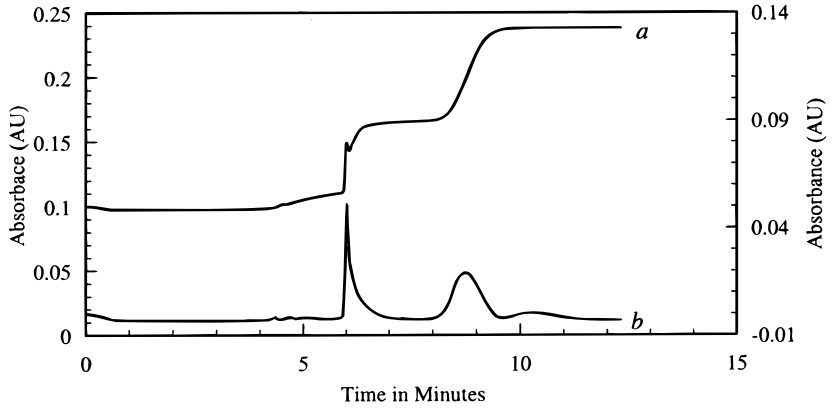

Figure 1. Electropherograms for a mixture of BSA $(1.85 \mathrm{~g} / \mathrm{L})$ and NaPSS (ca. $0.1 \mathrm{~g} / \mathrm{L}$ ) of MW $=88 \mathrm{~K}$ in phosphate buffer of $I=0.05 \mathrm{M}$ and $\mathrm{pH}=6.7$ : (a) obtained by FACCE; (b) obtained by injecting complex sample into protein-free buffer.

measured in this manner, since these complexes undergo appreciable dissociation in the protein-free Buffer. ${ }^{25}$

The concentration of bound protein in each sample was obtained by subtracting the free protein concentration determined via FACCE from the total protein concentration. The binding density, $v$, i.e., the number of protein molecules bound per polymer residue, is then calculated as

$$
v=\frac{[\text { Protein }]_{\text {bound }}}{[\mathrm{NaPSS}]_{\text {total }}} \frac{m_{0}}{M_{\text {pro }}}
$$

and the molecular weight of the complex, $M_{x}$, ean be calculated as

$$
M_{x}=\frac{[\text { Protein }]_{\text {bound }}+[\mathrm{NaPSS}]_{\text {total }}}{[\mathrm{NaPSS}]_{\text {total }}} M_{\mathrm{NaPSS}}
$$

where $m_{0}=206$ is the molar mass of the NaPSS monomer unit, $M_{\text {pro }}$ is the $M_{\mathrm{W}}$ of the protein, and $M_{\mathrm{NaPSS}}$ is the $M_{\mathrm{W}}$ of NaPSS. All concentration terms in eqs 5 and 6 bear units of g/L. Computer graphing and fitting of the binding data according to eqs 3 and 4 were done via DeltaGraph 4.0 dynamically linked to Excel 7.0.

\section{Results and Discussion}

a. Conformation of NaPSS in the Complexes. The doublelogarithmic plots of $D_{\mathrm{app}}$ versus $M_{\mathrm{w}}$ shown in Figure 2 for NaPSS and its complexes with BSA and $\beta$-lactoglobulin, respectively, correspond to the relationship

$$
D_{\text {app }}=K M^{a}
$$

where $M$ represents the $M_{\mathrm{w}}$ of NaPSS or the complexes. The value of $a$ in eq 7 is a measure of the expansion of the polymer or complex in solution, ${ }^{35}$ and $K$ reflects its density. The value of $a$ for NaPSS in Table 1 is in poor agreement with the $a$ value ranging from -0.60 to -0.66 reported by Wang and $\mathrm{Yu}^{24}$ at similar ionic strength. Since the $a$ value for NaPSS is dependent on its degree of sulfonation, ${ }^{36}$ and commercial samples of NaPSS vary somewhat in degree of sulfonation, ${ }^{29,36}$ it is difficult to compare results obtained by different workers. However, the important point here is the proximity of the values of $a$ found for NaPSS versus its complexes with the two proteins. The values of $K$ are in the order BSA/NaPSS $>\beta$-lactoglobulin/ NaPSS $>$ NaPSS, indicating the density of the complex is larger than that of protein-free NaPSS and the complex formed with the more massive protein possesses higher density.

The similarity of the structures of the complexes to that of the protein-free NaPSS prompted us to evaluate the draining

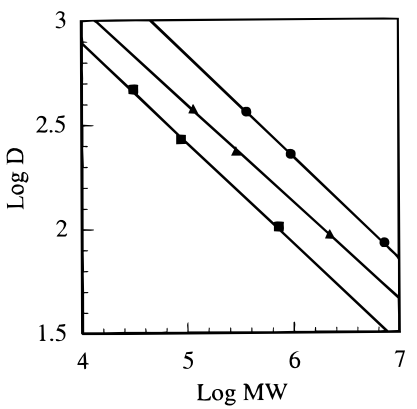

Figure 2. Correlation of diffusion coefficients $\left(\times 10^{-13} \mathrm{~m}^{2} / \mathrm{s}\right)$ and molecular weights for NaPSS (ם) and its complexes with BSA (0) and $\beta$-lactoglobulin $(\boldsymbol{\Delta})$ in phosphate buffer of $\mathrm{pH}=6.7$ and $I=0.05$ M. Symbols are experimental data. Lines are best-fit lines.

TABLE 1: Best-Fit Parmeters for Eq 7

\begin{tabular}{lcc}
\multicolumn{1}{c}{ parameter } & $a$ & $K\left(10^{-13} \mathrm{~m}^{2} / \mathrm{s}\right)$ \\
\hline NaPSS & -0.5 & $6.7 \times 10^{4}$ \\
BSA/NaPSS complex & -0.5 & $1.8 \times 10^{5}$ \\
$\beta$-lactoglobulin/NaPSS complex & -0.5 & $8.6 \times 10^{4}$
\end{tabular}

properties of the complexes. As the polymer is free-draining by itself in electrophoresis, it is interesting to evaluate whether the complexes, with similar structures, are free-draining as well.

b. Free-Draining Model for Complex Structure. Evaluation of the free-draining model described by eq 1 requires comparison of measured mobilities with calculated ones, based on known effective charges and frictional coefficients of proteins and polyelectrolytes. It is known that the effective charge of a protein in electrophoresis, $q_{\text {pro, }}$, is smaller than the net charge calculated from protein sequence information or $\mathrm{pH}$ titration data. ${ }^{38-40}$ This difference mainly arises from specific ionbinding, which is also responsible for the effect of the type of supporting electrolyte on the isoelectric point of proteins observed by moving boundary electrophoresis. ${ }^{41}$ In the absence of a direct measurement of $q_{\text {pro }}$, two indirect approaches have been proposed. By derivatizing a protein to form a series of protein charge ladders, Gao et al. ${ }^{42,43}$ were able to obtain the effective charge of the underivatized protein by extrapolation of mobility measurements. However, this method may not be applicable to high molecular weight proteins, as the resulting charge ladders may not be resolvable by CE. ${ }^{42}$ The effective protein charge can also be calculated by ${ }^{38}$

$$
\mu_{\text {pro }}=\frac{q_{\text {pro }}}{f_{\text {pro }}}
$$

which in combination with the Einstein equation, $f=k T / D_{\mathrm{T}}$, gives $^{44}$

$$
\mu_{\mathrm{pro}}=\frac{q_{\mathrm{pro}} D_{\mathrm{T}}}{k T}
$$

where $D_{\mathrm{T}}$ is the translational diffusion coeffficient $\left(\mathrm{m}^{2} / \mathrm{s}\right), k$ Boltzmann's constant $(\mathrm{J} / \mathrm{K})$, and $T$ the temperature $(\mathrm{K})$. The application of eq 9 to charged particles assumes that hydrodynamic friction in translational diffusion is similar to that in electrophoresis and that the value of $D_{\mathrm{T}}$ is not affected by electrostatic interactions.

Previous studies on $\mathrm{BSA}^{45,46}$ suggest that the apparent diffusion coefficient, $D_{\text {app }}$, measured by DLS is relatively insensitive to $\mathrm{pH}$ and ionic strength under the solvent conditions for our experiments. The concentration of BSA $(0.85 \mathrm{~g} / \mathrm{L})$ used in our DLS measurements is sufficiently low so that $D_{\text {app }}$ 
approaches its infinite dilution value. ${ }^{47-49}$ Hence, we assume in this work that the effects of electrostatic interaction on $D_{\text {app }}$ are insignificant for BSA, and $D_{\mathrm{app}} \approx D_{\mathrm{T}}$. Furthermore, since proteins are nondraining in both translational diffusion and electrophoresis, it is reasonable to expect that the friction experienced by proteins in both situations is similar, even though counterions move along with macroions in translational diffusion and move against them in electrophoresis. Therefore, to a first approximation, eq 9 is suitable for proteins, at least for BSA. However eq 9 is not applicable to polyelectrolytes. This is because the values of $D_{\text {app }}$ from DLS for flexible-chain polyelectrolytes are strongly affected by electrostatic interactions (i.e., in general $D_{\text {app }} \neq D_{\mathrm{T}}$ ), ${ }^{31}$ and more importantly, the frictional resistance in translational diffusion is very different from that in electrophoresis for flexible-chain polyelectrolytes, since they are nondraining in the former case and free-draining in the latter. ${ }^{50}$

Not being able to determine the effective electrophoretic charges on the polyelectrolyte $\left(q_{\mathrm{p}}\right.$ in eq 1$)$ experimentally, we resort to existing theories. According to Manning's counterion condensation theory, ${ }^{51}$ counterions condense on a polyelectrolyte chain when its linear charge density, $\xi$, exceeds the reciprocal of the valence of counterions in solution. This prediction has been confirmed inter alia by $\mathrm{CE}$ measurements of polyelectrolytes. ${ }^{52}$ Manning suggests that the mobility of polyelectrolytes in electrophoresis is reduced not only by the condensed counterions but also by an asymmetry effect from the distortion of ion atmosphere surrounding a polyelectrolyte in an electric field. ${ }^{53}$ Under our experimental conditions, the mobility calculated for NaPSS with consideration of the asymmetry effect is ca. $32 \%$ smaller than the result calculated without it (see Appendix). For proteins, however, the asymmetry effect is negligible. ${ }^{54}$ While one may easily perceive that the condensed counterions contribute to the effective charge of the polyelectrolyte, the connection between the asymmetry effect and the "effective charge" is less clear. To simplify our calculation, we collect all these mobility-diminishing effects in the effective charge of the polyelectrolyte. Therefore, we have

$$
\left|q_{\mathrm{p}}\right|=0.68 X_{\mathrm{p}} \xi^{-1}
$$

where $X_{\mathrm{p}}$ is the total number of monomer units in the polyelectrolyte and $\xi$ is the linear charge density of the polyelectrolyte. We also calculated the effective polyelectrolyte charge based on other theories describing the degree of counterion association. On the basis of an equilibrium twostate model for the counterion activity in a polyelectrolyte solution, Record et al. ${ }^{55}$ determined the extent of "thermodynamically associated counterions". If all such counterions were mobility-reducing counterions, then the effective absolute charge on a polyelectrolyte structural unit in the presence of univalent counterions would be $\mathrm{e}^{55}$

$$
\left|q_{\mathrm{p}}\right|=X_{\mathrm{p}}(2 \xi)^{-1}
$$

The same result is also reached upon consideration of the distribution of counterions around a polyion via the PoissonBoltzmann equation in cylindrical geometry. ${ }^{56}$ It is noted that the effective charge from eq $10 \mathrm{~b}$ is half of the charge calculated based on counterion condensation alone. ${ }^{51}$ For a comparison, we will include results of complex mobility calculation using expressions in both eq 10a and eq $10 \mathrm{~b}$.

To reduce the free-draining model of eq 1 to a more useful form based on the properties of the complex per polymer
TABLE 2: Calculated Frictional Coefficients and Experimentally Determined Parameters in $I=0.05 \mathrm{M}, \mathrm{pH}=$

\begin{tabular}{|c|c|c|c|}
\hline sample & $\begin{array}{l}\text { effective } \\
\text { charge }(e)\end{array}$ & $\begin{array}{c}\text { mobility } \\
\left(10^{-4} \mathrm{~cm}^{2} /(\mathrm{V} \cdot \mathrm{s})\right)\end{array}$ & $\begin{array}{c}\text { calculated frictional } \\
\text { coefficient } \\
\left(10^{4} \mathrm{~V} \cdot \mathrm{s} \cdot \mathrm{e} / \mathrm{cm}^{2}\right)\end{array}$ \\
\hline $\begin{array}{l}\text { NaPSS } \\
(\xi=2.84)\end{array}$ & $\begin{array}{l}-c \xi^{-1} \\
(c=0.68 \\
\quad \text { or } 0.50)\end{array}$ & -3.89 & $\begin{array}{l}f_{\mathrm{m}}=0.061(c=0.68) \\
f_{\mathrm{m}}=0.045(c=0.50)\end{array}$ \\
\hline $\begin{array}{l}\text { BSA } \\
\left(D_{\text {app }}=6.40 \times\right. \\
\left.10^{-11} \mathrm{~m}^{2} / \mathrm{s}\right) \\
\beta \text {-lactoglobulin }\end{array}$ & -4.90 & -1.22 & $f_{\text {pro }}=4.02$ \\
\hline $\begin{array}{c}\left(D_{\text {app }}=7.35 \times\right. \\
\left.10^{-11} \mathrm{~m}^{2} / \mathrm{s}\right)\end{array}$ & -4.34 & -1.24 & $f_{\text {pro }}=3.50$ \\
\hline
\end{tabular}
6.7 Phosphate Buffer at $25^{\circ} \mathrm{C}$

structural unit, we express the binding density, $v$, as

$$
v=\frac{N}{X_{\mathrm{p}}}
$$

Since polyions are free-draining in electrophoresis, we write $f_{\mathrm{p}}$ as

$$
f_{\mathrm{p}}=X_{\mathrm{p}} f_{\mathrm{m}}
$$

where $f_{\mathrm{m}}$ is the average contribution of each monomer to the total polymer frictional coefficient. Substitution of eqs 10-12 into eq 1 then yields the following relationship between complex mobility and complex composition:

$$
\mu_{x}=\frac{ \pm c \xi^{-1}+v q_{\mathrm{pro}}}{f_{\mathrm{m}}+v f_{\mathrm{pro}}}
$$

where $c$ is a constant with a value of either 0.68 (eq 10a) or 0.5 (eq 10b). Here, it is assumed that neither the polymer linear charge density, $\xi$, nor the net protein charge, $q_{\text {pro, }}$, changes upon complex formation. These assumptions are valid when the number of counterions released from the polyelectrolyte upon binding is only a small fraction of the condensed counterions or when the numbers of released cations and anions from the polyelectrolyte and the protein upon binding are approximately equal.

Table 2 contains theoretical values of the effective charge for NaPSS, the mobilities for proteins and NaPSS determined by $\mathrm{CE}$, and the effective protein charges, along with the frictional coefficients for proteins and NaPSS calculated using eqs 8 and 9 . The complex mobility can then be calculated by substituting the experimentally determined binding density into eq 13. For BSA/NaPSS complexes, results of measured and calculated mobilities along with the binding density data required in the calculation are listed in Table 3. Measured and calculated mobilities for BSA/NaPSS complexes are in remarkable agreement when "thermodynamically associated counterions" are used to calculate the polymer effective charge, while larger discrepancies exist when the polymer effective charge was calculated according to the counterion condensation and the asymmetry effect. However, due to the many approximations implicit in the free-draining model expressed as eq 13 and our simplified treatment of the asymmetry effect, the difference between the measured and calculated mobilities should not be used to differentiate between the theories on which the calculations were based. The mobilities of $\beta$-lactoglobulin/NaPSS complexes were calculated in similar ways and are listed in Table 4 along with the measured binding density. The dependence of calculated mobilities upon NaPSS $M_{\mathrm{w}}$ for both protein complexes is 
TABLE 3: Calculated and Measured Mobility for BSA/NaPSS Complexes in Phosphate Buffer of $I=0.05 \mathrm{M}$ and $\mathrm{pH}=6.7$

\begin{tabular}{|c|c|c|c|c|c|c|}
\hline $\begin{array}{l}\text { NaPSS } \\
\text { MW }\end{array}$ & $v \times 10^{2}$ & $\begin{array}{c}\text { measured complex } \\
\text { mobility }(\mu \mathrm{m} \cdot \mathrm{cm} /(\mathrm{V} \cdot \mathrm{s}))\end{array}$ & $\begin{array}{c}\text { calculated }{ }^{a} \text { complex } \\
\text { mobility }(\mu \mathrm{m} \cdot \mathrm{cm} /(\mathrm{V} \cdot \mathrm{s}))\end{array}$ & $\begin{array}{l}\text { relative difference }{ }^{a} \\
(\%)\end{array}$ & $\begin{array}{c}\text { calculated }{ }^{b} \text { complex } \\
\text { mobility }(\mu \mathrm{m} \bullet \mathrm{cm} /(\mathrm{V} \cdot \mathrm{s}))\end{array}$ & $\begin{array}{c}\text { relative difference }{ }^{b} \\
(\%)\end{array}$ \\
\hline 5400 & 4.56 & -1.76 & -1.75 & 0.07 & -1.89 & 8 \\
\hline 31000 & 3.37 & -1.83 & -1.89 & 3 & -2.06 & 12 \\
\hline 88000 & 3.07 & -1.90 & -1.94 & 2 & -2.11 & 11 \\
\hline 710000 & 2.83 & -1.92 & -1.98 & 3 & -2.16 & 12 \\
\hline
\end{tabular}

${ }^{a}$ Effective charge on each polymer structual unit is based on eq $10 \mathrm{~b} .{ }^{b}$ Effective charge on each polymer structual unit is based on eq 10a.

TABLE 4: Calculated Mobility for $\beta$-Lactoglobulin/NaPSS Complexes in Phosphate Buffier of $I=0.05 \mathrm{M}$ and $\mathrm{pH}=6.7$

\begin{tabular}{rccc}
\hline \multicolumn{1}{c}{$\begin{array}{c}\text { NaPSS } \\
\text { MW }\end{array}$} & $v \times 10^{2}$ & $\begin{array}{c}\text { calculated }^{a} \text { complex } \\
\text { mobility }(\mu \mathrm{m} \cdot \mathrm{cm} /(\mathrm{V} \cdot \mathrm{s}))\end{array}$ & $\begin{array}{c}\text { calculated } \\
\text { mobility }^{b}(\mu \mathrm{m} \cdot \mathrm{cm} /(\mathrm{V} \cdot \mathrm{s}))\end{array}$ \\
\hline 5400 & 1.12 & -2.66 & -2.86 \\
31000 & 2.40 & -2.17 & -2.36 \\
88000 & 2.50 & -2.14 & -2.33 \\
710000 & 2.50 & -2.14 & -2.33
\end{tabular}

${ }^{a}$ Effective charge on each polymer structual unit is based on eq $10 \mathrm{~b}$. ${ }^{b}$ Effective charge on each polymer structual unit is based on eq 10a.

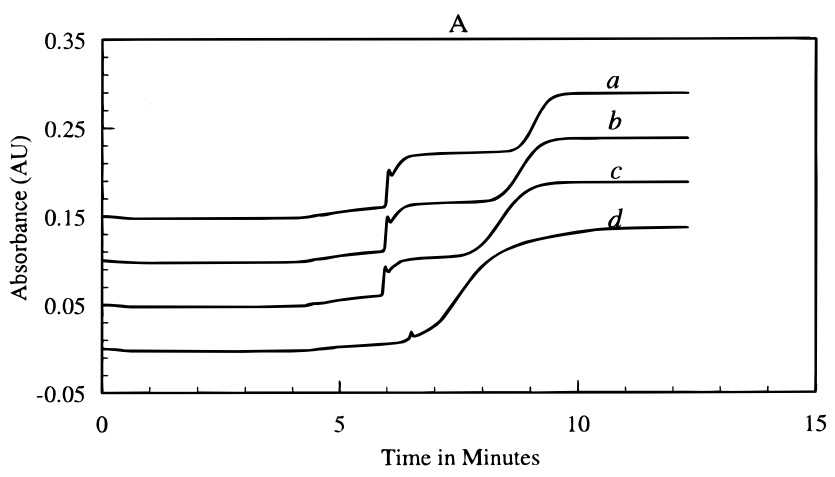

B

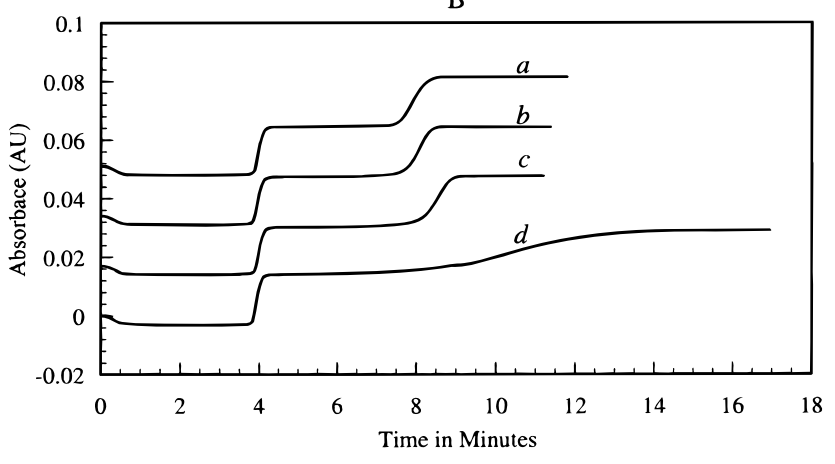

Figure 3. FACCE electropherograms for NaPSS (ca. $0.1 \mathrm{~g} / \mathrm{L}$ ) of various MW's complexing with (A) BSA $(1.85 \mathrm{~g} / \mathrm{L})$, (B) $\beta$-lactoglobulin $(0.83$ $\mathrm{g} / \mathrm{L}$ ) in phosphate buffer of $I=0.05$ and $\mathrm{pH}=6.7$ : (a) $\mathrm{MW}_{\mathrm{NaPSS}}=$ $710 \mathrm{~K}$, (b) $\mathrm{MW}_{\mathrm{NaPSS}}=88 \mathrm{~K}$, (c) $\mathrm{MW}_{\mathrm{NaPSS}}=31 \mathrm{~K}$, (d) $\mathrm{MW}_{\mathrm{NaPSS}}=5.4 \mathrm{~K}$.

in qualitative agreement with the observation in Figure 3. The stacked FACCE electropherograms indicate that as the $M_{\mathrm{w}}$ of NaPSS decreases, complex plateaus (second plateaus) appear sooner for BSA/NaPSS complexes (Figure 3A) and later for $\beta$-lactoglobulin/NaPSS complexes (Figure 3B).

The apparent success of the free-draining model prompted us to further consider the correlation of the mobility and the composition of a free-draining complex. The plot of eq 13 with $c=0.5$ in Figure 4 shows that complex mobility is most sensitive to composition change in the low binding density region. As binding density increases, its effect on complex mobility decreases. The change in complex mobility as a function of change in binding density can be described mathematically. Upon differentiation of eq 13 , for polyanions, we have

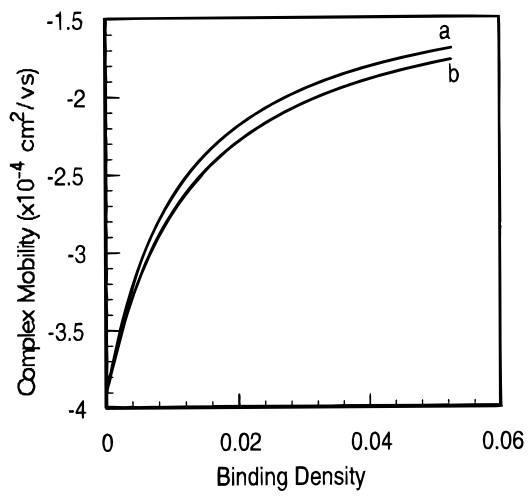

Figure 4. Complex mobility as a function of binding density for BSA (a) and $\beta$-lactoglobulin (b) complexing with NaPSS in phosphate of $I$ $=0.05 \mathrm{M}$ and $\mathrm{pH}=6.7$.

$$
\frac{\mathrm{d} \mu}{\mathrm{d} \nu}=\frac{q_{\mathrm{pro}} f_{\mathrm{m}}+c \xi^{-1} f_{\mathrm{pro}}}{\left(f_{\mathrm{m}}+v f_{\mathrm{pro}}\right)^{2}}
$$

Thus, the change in complex mobility, $\Delta \mu$, is a function of the change in binding density, $\Delta v$, in the form

$$
\Delta \mu=\left(\frac{q_{\mathrm{pro}} f_{\mathrm{m}}+c \xi^{-1} f_{\mathrm{pro}}}{\left(f_{\mathrm{m}}+v f_{\mathrm{pro}}\right)^{2}}\right) \Delta v
$$

Substituting values for BSA from Table 2 and $c=0.5$ into eq 15 , we find the value of the term in brackets varies from the maximum of $458\left(10^{-4} \mathrm{~cm}^{2} / \mathrm{Vs}\right)$ at $v=0$, to $128\left(10^{-4} \mathrm{~cm}^{2} /\right.$ $\mathrm{Vs})$ at $v=0.01$, to $15\left(10^{-4} \mathrm{~cm}^{2} / \mathrm{Vs}\right)$ at $v=0.05$. Since the accuracy of the mobility measurement in this work is \pm 0.01 $\left(10^{-4} \mathrm{~cm}^{2} / \mathrm{Vs}\right)$ and that of the binding density is \pm 0.0001 , the sensitivity of complex mobility measurements is comparable to or better than concentration measurements for characterizing complex composition when the binding density is less than 0.01 . Therefore, complex mobility measurements in the low binding density region provide an alternative to other methods that suffer from greater noise, especially when the binding data in the low binding density region determine the intrinsic binding constants. ${ }^{12}$

c. Analysis of Binding via McGhee-von Hippel Model. The retention of polyelectrolyte conformation upon complex formation and the free-draining behavior of the complex suggest that multiple attachments of remote segments of NaPSS to a single protein are unlikely, so that the binding between proteins and NaPSS may be treated approximately by the McGheevon Hippel model ${ }^{12}$ for large ligands binding to a onedimensional lattice with overlapping binding sites. Fitting of the binding isotherms in Figure 5 according to eqs 3 and 4 leads to an average binding site size of 17 monomer residues for $\beta$-lactoglobulin and 17-24 monomer residues for BSA, depending upon solution conditions (Table 5). If the number of monomer residues occluded by a bound protein is solely based on the one-dimensional lengths of protein and polymer chain, then, from the hydrodynamic diameters of 7.0 and $5.4 \mathrm{~nm}$ for 


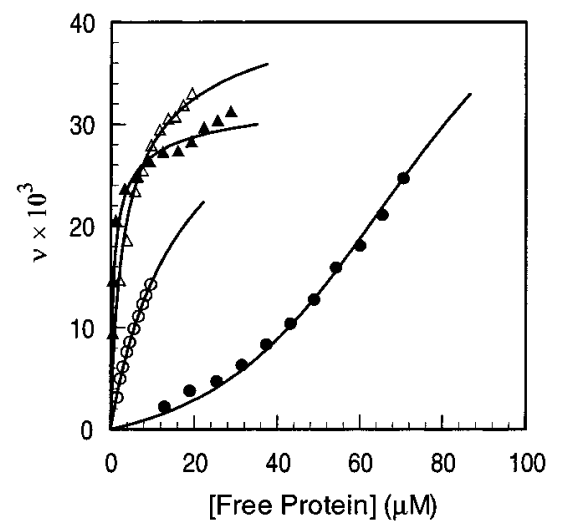

Figure 5. Binding density as a function of free protein concentration. Symbols are experimental data, lines are best-fit theoretical curves according to eqs 3 and 4 with the parameter values in Table 5: $(\triangle)$ BSA/NaPSS, $\mathrm{pH}=6.7 ; I=0.05 \mathrm{M} ;(\boldsymbol{\Delta}) \mathrm{BSA} / \mathrm{NaPSS}, \mathrm{pH}=7.5 ; I=$ $0.1 \mathrm{M}$; (O) $\beta$-lactoglobulin/NaPSS, $\mathrm{pH}=6.7 ; I=0.05 \mathrm{M}$; $\beta$-lactoglobulin-NaPSS, $\mathrm{pH}=7.5 ; I=0.1 \mathrm{M}$.

TABLE 5: Values of Parameters Used to Fit Binding Isotherms in Figure 5

\begin{tabular}{lll}
\hline \multicolumn{1}{c}{ sample } & \multicolumn{1}{c}{$\mathrm{pH}=6.7 ;$} & \multicolumn{1}{c}{$\mathrm{pH}=7.5 ;$} \\
$\beta$-lactoglobulin/NaPSS & $n=17$ & \multicolumn{1}{c}{$I=0.1 \mathrm{M}$} \\
& $K_{\mathrm{b}}=(2.5 \pm 0.3) \times$ & $n=17$ \\
& $10^{3} \mathrm{M}^{-1}$ & $K_{\mathrm{b}}=(9.4 \pm 0.3) \times$ \\
& $\omega=3$ & $10 \mathrm{M}^{-1}$ \\
BSA/NaPSS & $n=17$ & $\omega=128$ \\
& $K_{\mathrm{b}}=(1.5 \pm 0.3) \times$ & $n=24$ \\
& $10^{4} \mathrm{M}^{-1}$ & $10^{4} \mathrm{M}^{-1}$ \\
& $(\omega=0)^{a}$ & $(\omega=0)^{a}$
\end{tabular}

${ }^{a}$ The theoretical curve is not sensitive to $\omega$ for $\omega<1$.

SCHEME 1: Comparison of Binding Models: (a) OneDimensional Approach of Ligands to Aligned Linear Binding Sites in McGhee-von Hippel Model: (b) Multidirectional Approach of Ligands to Binding Sites Assuming Various Orientations in Real Systems
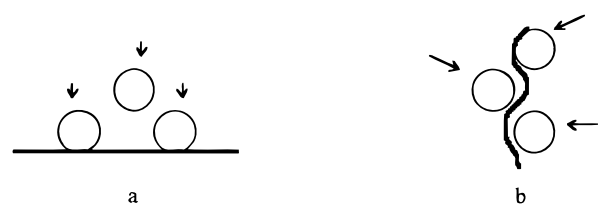

BSA and $\beta$-lactoglobulin, respectively, and from the spacing of $2.5 \AA$ between adjacent monomer residues, we calculate binding site sizes of 28 monomer residues for BSA and 22 monomer residues for $\beta$-lactoglobulin. However, such calculations are based on the assumption that all binding sites are aligned in one direction and can only be approached from one direction (Scheme 1a), whereas binding sites on a linear polyelectrolyte have different orientations and can be accessed from various directions (Scheme 1b). Therefore, it is possible that the true size of the binding sites is smaller than such calculated ones. In addition, in the case of anticooperative binding, anticooperativity maybe imbedded in the binding site size, thus making the apparent size larger than the real one. In this aspect, we want to mention that, in our curve-fitting process for BSA/NaPSS binding data, fitted curves are much less sensitive to the changes in $\omega$ than to the changes in $n$.

Cooperativity in complex formation is reflected in both the shape of the binding isotherm and the fitting parameters. The shape of isotherms in Figure 5 suggests that the binding of BSA to NaPSS at $\mathrm{pH}=6.7$ and $I=0.05 \mathrm{M}$ is anticooperative, whereas the binding of $\beta$-lactoglobulin to NaPSS is not, as

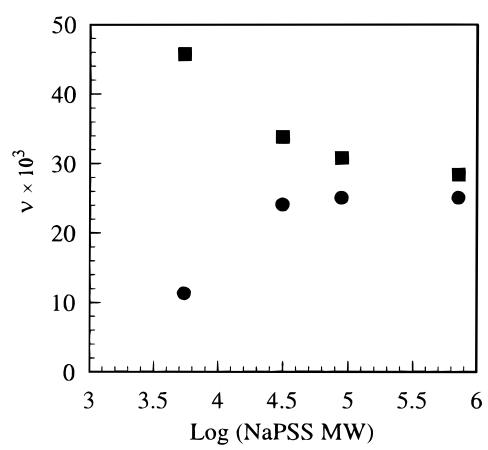

Figure 6. Binding density as a function of MW of NaPSS for BSA/ NaPSS samples (ם) and $\beta$-lactoglobulin/NaPSS samples $(\bullet)$.

confirmed by $\omega<1$ (anticooperative) for the former and $\omega=$ 3 (slightly cooperative) for the latter. The difference in binding cooperativity for BSA versus $\beta$-lactoglobulin is more discernible at $\mathrm{pH}=7.5$ and $I=0.1 \mathrm{M}$ than at $\mathrm{pH}=6.7$ and $I=0.05 \mathrm{M}$, with BSA binding being anticooperative $(\omega<1)$ and $\beta$-lactoglobulin binding being very cooperative $(\omega=128)$. These observations can be correlated with the dependence of binding density upon NaPSS $M_{\mathrm{w}}$ presented in the last section.

The values of intrinsic binding constants are quantitative measures of binding affinity. Under the same solvent condition, BSA shows stronger binding than $\beta$-lactoglobulin. The dependence of binding affinity on solvent condition is also different for the two proteins. As $\mathrm{pH}$ and ionic strength increase from one buffer to another, $K_{\mathrm{b}}$ for BSA shows a 4-fold increase, whereas $K_{\mathrm{b}}$ for $\beta$-lactoglobulin exhibits a decrease of nearly 2 orders of magnitude (Table 5). This difference is probably due to the different balance between electrostatic repulsion and hydrophobic interaction, as it has been observed ${ }^{57}$ that BSA is more hydrophobic than $\beta$-lactoglobulin, and the hydrophobicity of the former increases with $\mathrm{pH}$ while the hydrophobicity of the latter is relatively constant in the two buffers used. The question of hydrophobic interactions involving NaPSS will be the subject of a separate investigation.

d. Dependence of Binding Density upon $M_{\mathrm{w}}$ of NaPSS and Binding Cooperativity. Results of binding dependence on NaPSS $M_{\mathrm{w}}$ are shown in Figure 6. Binding density of BSA decreases as the polymer chain length increases, and the opposite was observed for $\beta$-lactoglobulin. Such a difference in binding density dependence on polymer chain length agrees with the difference in binding cooperativities between the two proteins shown in the previous section. According to Epstein, ${ }^{28}$ as the lattice length or molecular weight of a linear polyelectrolyte increases, the binding density approaches an asymptotic limit, but from opposite directions for anticooperative versus cooperative systems. This is because a longer polymer chain has a higher fraction of adjacent binding sites, leading to stronger cooperativity effects. Thus as polyelectrolyte chain length increases, binding density increases for cooperative systems and decreases for anticooperative systems. These results suggest that bound proteins are likely to be discrete in BSA/NaPSS complexes, but contiguous in $\beta$-lactoglobulin/NaPSS complexes. However, the origin of the binding cooperativity difference for the two proteins is not clear.

\section{Conclusions}

From dynamic light scattering and capillary electrophoresis measurements of bovine serum albumin and $\beta$-lactoglobulin complexing with sodium polystyrenesulfonate, we have found that the conformations of these complexes are similar to those of the protein-free polyelectrolyte. In addition, our results are 
consistent with a free-draining model for the complexes. The fitting parameters of the binding isotherms and the effect of the polyelectrolyte molecular weight upon binding density indicate that the binding of bovine serum albumin is anticooperative, while the binding of $\beta$-lactoglobulin is cooperative.

Acknowledgment. We thank Prof. G. S. Manning of Rutgers University for helpful discussions and comments. We are also indebted to Prof. Y. Morishima of Osaka University for his suggestion. This work was supported by Grant DMR 9619722 from the National Science Foundation. J.Y.G. acknowledges a fellowship from Purdue Research Foundation.

\section{Appendix}

The asymmetry effect on the mobility of NaPSS in phosphate buffer of $I=0.05 \mathrm{M}$ was estimated based on the calculation for the symmetric electrolyte $\mathrm{NaH}_{2} \mathrm{PO}_{4}$ according to ref 53. All equation numbers and parameters cited in the following are from that reference.

With $\alpha=1$ for symmetric electrolytes and $U=3.89 \mu \mathrm{m}$. $\mathrm{cm} /(\mathrm{V} \cdot \mathrm{s})$ determined experimentally, $\beta$ can be calculated by simultaneously solving eq 35 and eq 38 . Values of parameters used in our calculations are

$$
v_{1}=1 ; \quad v_{2}=1 ; \quad z_{1}=1 ; \quad z_{2}=-1
$$

and the values of equivalent ionic conductivity at infinite dilution $\operatorname{are}^{58}$

$\mathrm{Na}^{+}: \quad \lambda_{1}=50.08 \times 10^{-4} \mathrm{~m}^{2} \cdot \mathrm{S} / \mathrm{mol}$

$\mathrm{H}_{2} \mathrm{PO}_{4}^{-}: \quad \lambda_{2}=33.00 \times 10^{-4} \mathrm{~m}^{2} \cdot \mathrm{S} / \mathrm{mol}$

Therefore, $\beta=1.464$. The asymmetry effect according to eq 35 is

$$
\frac{\alpha}{\beta}=\frac{1}{1.464} \approx 0.68
$$

In other words, the asymmetry effect reduces the mobility of NaPSS by ca. $32 \%$.

\section{References and Notes}

(1) Record, M. T., Jr.; Anderson, C. F.; Lohman, T. M. Q. Rev. Biophys. 1978, 11, 103 .

(2) von Hippel, P. H.; Bear, D. G.; Morgan, W. D.; McSwiggen, J. A. Annu. Rev. Biochem. 1984, 53, 389.

(3) Wang, Y.; Gao, J. Y.; Dubin, P. L. Biotechnol. Prog. 1996, 12, 356.

(4) Margolin, A.; Sheratyuk, S. F.; Izumrudov, V. A.; Zezin, A. B.; Kabanov, V. A. Eur. J. Biochem. 1985, 146, 625.

(5) Xia, J.; Dubin, P. L. In Macromolecular Complexes in Chemistry and Biology; Dubin, P. L., Davis, R. M., Schultz, D., Thies, C., Eds.; Springer-Verlag: Berlin, 1994; Chapter 15

(6) Regelson, W. Interferon 1970, 6, 353.

(7) Ottenbrite, R. M.; Kaplan, A. M. Ann. New York Acad. Sci. 1985 $446,160$.

(8) Manning, G. S. Q. Rev. Biophys. 1978, 11, 179

(9) (a) Record, M. T., Jr.; Ha, J.-H.; Fisher, M. A. Methods Enzymol. 1991, 208, 291. (b) Rouzina, I. F.; Bloomfield, V. A. J. Phys. Chem. 1996 100, 4292. (c) Rouzina, I. F.; Bloomfield, V. A. J. Phys. Chem. 1996, 100, 4305.

(10) (a) Epstein, I. R. Biopolymers 1979, 18, 2037. (b) Reiter, J.; Epstein I. R. Biopolymers 1990, 29, 543

(11) Muthukumar, M. J. Chem. Phys. 1995, 103, 4723.

(12) (a) McGhee, J. D.; von Hippel, P. H. J. Mol. Biol. 1974, 86, 469. (b) McGhee, J. D.; von Hippel, P. H. J. Mol. Biol. 1976, 103, 679.
(13) (a) Schumaker, M. F.; Epstein, I. Biopolymers 1990, 29, 1331. (b) Woodbery, C. P. Biopolymers 1981, 20, 2225. (c) Schwarz, G. Biophys. Chem. 1977, 6, 65

(14) Xia, J.; Dubin, P. L.; Dautzenberg, H. Langmuir 1993, 9, 2015.

(15) Xia, J.; Dubin, P. L.; Kim, Y.; Muhoberac, B. B.; Klimkowski, V. J. J. Phys. Chem. 1993, 97, 4528.

(16) Park, J. M.; Muhoberac, B. B.; Dubin, P. L.; Xia, J. Macromolecules $1992,25,290$

(17) Li, Y.; Mattison, K. W.; Dubin, P. L.; Havel, H. A.; Edwards, S L. Biopolymers 1996, 38, 527.

(18) Mattison, K. W.; Brittain, I. J.; Dubin, P. L. Biotechnol. Prog. 1995, 11,632

(19) Xia, J.; Dubin, P. L.; Morishima, Y.; Sato, T.; Muhoberac, B. B. Biopolymers 1995, 35, 411

(20) Tsuboi, A.; Izumi, T.; Hirata, M.; Xia, J.; Dubin, P. L.; Kokufuta, E. Langmuir 1996, 12, 6295 .

(21) Kuramoto, N.; Sakamoto, M.; Komiyama, J.; Iijima, T. Makromol. Chem. 1984, 185, 1419.

(22) Strege, M. A.; Dubin, P. L.; West, J. S.; Flinta, C. D. In Protein Purification: From Molecular Mechanism to Large-Scale Process; Ladisch, M., Wilson, R. C., Painton, C. C., Builder, S. E., Eds.; American Chemical Society: Washington, DC, 1990; Chapter 5.

(23) Xia, J.; Dubin, P. L. J. Chromatogr. A 1994, 667, 311

(24) Wang, L.; Yu, H. Macromolecules 1988, 21, 3498.

(25) Gao, J. Y.; Dubin, P. L.; Muhoberac, B. B. Anal. Chem. 1997, 69, 2945.

(26) Wyman, J.; Gill, S. J. Binding and Linkage: Functional Chemistry of Biological Macromolecules; University Science Books: Mill Valley, CA, 1990 .

(27) Kowalczykowski, S. C.; Paul, L. S.; Lonberg, N.; Newport, J. W.; McSwiggen, J. A.; von Hippel, P. H. Biochemistry 1986, 25, 1226.

(28) Epstein, I. R. Biophys. Chem. 1978, 8, 327.

(29) Yamanaka, J.; Matsuoka, H.; Kitano, H.; Hasegawa, M.; Ise, N. J. Am. Chem. Soc. 1990, 112, 587.

(30) Fasman, G. D., Ed. Practical Handbook of Biochemistry and Molecular Biology; CRC Press: Boca Raton, FL, 1990.

(31) Schmitz, K. S. An Introduction to Dynamic Light Scattering by Macromolecules; Academic Press: New York, 1990.

(32) Jorgenson, J. W.; DeArman Lukacs, K. Anal. Chem. 1981, 53, 1298

(33) Rose, D. J. Anal. Chem. 1993, 65, 3545.

(34) Chu, Y.-H.; Lees, W. J.; Stassinopoulos, A.; Walsh, C. T. Biochemistry 1994, 33, 10616.

(35) Yamakawa, H. Modern Theory of Polymer Solutions; Harper \& Row: New York, 1971.

(36) Molyneux, P. Water Soluble Synthetic Polymers, Vol. II; CRC Press: Boca Raton, FL, 1984; p 45.

(37) Brandrup, J., Immergut, E. H., Eds. Polymer Handbook; Wiley: New York, 1989.

(38) Hiemenz, P. C. Principles of Colloid and Surface Chemistry; Dekker: New York, 1986

(39) Compton, B. J.; O’Grady, E. A. Anal. Chem. 1991, 63, 2597

(40) Micinski, S.; Grønvald, M.; Compton, B. J. Methods Enzymol. 1996, $270,342$.

(41) Cann, J. R. Electrophoresis. In Physical Principles of Technology in Protein Chemistry; Leach, S. J., Ed.; Academic Press: New York, 1969 Part A, pp 369-450

(42) Gao, J.; Gomez, F. A.; Härter, R; Whitesides, G. M. Proc. Natl Acad. Sci. U.S.A. 1994, 91, 12027.

(43) Gao, J.; Whitesides, G. M. Anal. Chem. 1997, 69, 575.

(44) Abramson, H. A.; Moyer, L. S.; Gorin, M. H. Electrophoresis of Proteins; Hafner Publishing: New York, 1964.

(45) Raj, T.; Flygare, W. H. Biochemsitry 1974, 13, 3336

(46) Doherty, P.; Benedek, G. B. J. Chem. Phys. 1974, 61, 5426.

(47) Neal, D. G.; Purich, D.; Cannell, D. S. J. Chem. Phys. 1984, 80, 3469.

(48) Harvey, J. D : Geddes, R. Wills, P. R. Biopolymers 1979, 18, 2249.

(49) Oh, Y. S.; Johnson, C. S., Jr. J. Chem. Phys. 1981, 74, 2717

(50) Manning, G. S. J. Phys. Chem. 1980, 84, 1059

(51) Manning, G. S. Acc. Chem. Res. 1979, 12, 443.

(52) Gao, J. Y.; Dubin, P. L.; Sato, T.; Morishima, Y. J. Chromatogr. A 1997, 766, 233.

(53) Manning, G. S. J. Phys. Chem. 1981, 85, 1506

(54) Overbeek, J. T. G.; Wiersema, P. H. in Electrophoresis: Theory, Methods, and Applications; Bier, M., Ed.; Academic Press: New York, 1967; Vol. II, Chapter 1.

(55) Record, M. T., Jr.; Lohman, T. M.; de Haseth, P. J. Mol. Biol. 1976, 107, 145

(56) Kotin, L.; Nagasawa, M. J. Chem. Phys. 1962, 36, 873

(57) Kato, A.; Matsuda, T.; Matsudomi, N.; Kobayashi, K. J Agric. Food Chem. 1984, 32, 284

(58) Lide, D. R., Ed. CRC Handbook of Chemistry and Physics, 72nd ed.; CRC Press: Boston, 1991 